\title{
The buffy coat method: a tool for detection of blood parasites without staining procedures
}

\author{
Carolina R. F. Chagas* ${ }^{*}$, Rasa Binkienè, Mikas Ilgūnas, Tatjana lezhova and Gediminas Valkiūnas
}

\begin{abstract}
Background: Blood parasites belonging to the Apicomplexa, Trypanosomatidae and Filarioidea are widespread in birds and have been studied extensively. Microscopical examination (ME) of stained blood films remains the gold standard method for the detection of these infections in birds, particularly because co-infections predominate in wildlife. None of the available molecular tools can detect all co-infections at the same time, but ME provides opportunities for this to be achieved. However, fixation, drying and staining of blood films as well as their ME are relatively time-consuming. This limits the detection of infected hosts during fieldwork when captured animals should be released soon after sampling. It is an obstacle for quick selection of donor hosts for parasite experimental, histological and other investigations in the field. This study modified, tested and described the buffy coat method (BCM) for quick diagnostics ( $20 \mathrm{~min} / \mathrm{sample}$ ) of avian blood parasites.

Methods: Blood of 345 birds belonging to 42 species was collected, and each sample was examined using ME of stained blood films and the buffy coat, which was examined after centrifugation in capillary tubes and after being transferred to objective glass slides. Parasite detection using these methods was compared using sensitivity, specificity, positive and negative predictive values and Cohen's kappa index.

Results: Haemoproteus, Leucocytozoon, Plasmodium, microfilariae, Trypanosoma and Lankesterella parasites were detected. BCM had a high sensitivity (>90\%) and specificity (>90\%) for detection of Haemoproteus and microfilariae infections. It was of moderate sensitivity (57\%) and high specificity (>90\%) for Lankesterella infections, but of low sensitivity (20\%) and high specificity (>90\%) for Leucocytozoon infections. Trypanosoma and Plasmodium parasites were detected only by BCM and ME, respectively. According to Cohen's kappa index, the agreement between two diagnostic tools was substantial for Haemoproteus (0.80), moderate for Lankesterella (0.46) and fair for microfilariae and Leucocytozoon (0.28) infections.
\end{abstract}

Conclusions: BCM is sensitive and recommended as a quick and reliable tool to detect Haemoproteus, Trypanosoma and microfilariae parasites during fieldwork. However, it is not suitable for detection of species of Leucocytozoon and Plasmodium. BCM is a useful tool for diagnostics of blood parasite co-infections. Its application might be extended to studies of blood parasites in other vertebrates during field studies.

Keywords: Blood parasites, Parasite diagnostics, Birds, Haemoproteus, Plasmodium, Leucocytozoon, Trypanosoma, Lankesterella, Microfilaria, Fieldwork

*Correspondence: crfchagas@gmail.com

Nature Research Centre, Akademijos 2, 08412 Vilnius, Lithuania

\section{Background}

Blood parasites belonging to the Apicomplexa, Trypanosomatidae, and microfilariae of filariid nematodes (Filarioidea) are widespread and have been extensively reported in different bird groups all over the world [1-10]. Blood parasites also infect fishes [11, 12], amphibians [13, 14],

(c) The Author(s) 2020. This article is licensed under a Creative Commons Attribution 4.0 International License, which permits use, sharing, adaptation, distribution and reproduction in any medium or format, as long as you give appropriate credit to the original author(s) and the source, provide a link to the Creative Commons licence, and indicate if changes were made. The images or other third party material in this article are included in the article's Creative Commons licence, unless indicated otherwise in a credit line to the material. If material is not included in the article's Creative Commons licence and your intended use is not permitted by statutory regulation or exceeds the permitted use, you will need to obtain permission directly from the copyright holder. To view a copy of this licence, visit http://creativeco mmons.org/licenses/by/4.0/. The Creative Commons Public Domain Dedication waiver (http://creativecommons.org/publicdomain/ zero/1.0/) applies to the data made available in this article, unless otherwise stated in a credit line to the data. 
reptiles [15] and mammals [16]. Nowadays, there are two main methods, which are broadly used to diagnose these infections: microscopic examination (ME) of blood films, usually stained with Giemsa; and polymerase chain reaction (PCR)-based testing [13, 17-29].

ME is useful to detect infections not only at relatively high levels of parasitemia, but also during light chronic infections [19]. It is also recommended for detection of co-infections, which predominate in wildlife [19, 30, 31]. Available PCR-based diagnostic protocols using general primers are often insensitive to detect co-infections of closely related blood parasites in many taxonomic groups [30-33]. Compared to the molecular protocols, blood film ME is cheaper, often faster and can be used even during fieldwork if access to relatively simple microscopic facilities is available. Additionally, molecular characterization of the majority of described blood parasite species remains to be developed; however, many of the species or genera can be distinguished using morphological characters of the blood stages. From this point of view, ME of stained blood films can still be considered as the gold standard method for blood parasite biodiversity research in wildlife. However, this methodology is rather time-consuming for field studies, and this creates obstacles in examining large numbers of animals at the study site in the wild. Additionally, it requires good quality blood films, which must be fixed, dried and stained properly to reach good results during microscopic diagnostics [19]. Proper staining and the subsequent ME procedures are often difficult to achieve during fieldwork, particularly in remote areas.

Several studies reported that PCR could potentially be more sensitive for detection of avian haemosporidian parasites, especially during light chronic infections $[17,18]$. However, professional microscopic examination can also be just as sensitive in detection of blood parasites. It is important to note that PCR-based techniques provide other valuable data in addition to simple parasite detection, particularly regarding parasite DNA sequence information, which can be used for populational genetics, phylogenetics, epidemiology, vector and other studies [10, 22, 23, 34-38]. Unfortunately, the application of PCR-based techniques is usually impossible during fieldwork due to strict requirements for materials and laboratory infrastructure. Moreover, molecular diagnostic procedures (DNA extraction, PCR, electrophoresis, DNA precipitation and sequencing) are also time-consuming, and depending on the PCR-based protocols applied, it usually takes several days to obtain sequence information.

Quick detection of infected animals in the wild is essential for collection of parasite donor-hosts, which are often needed for helminthology [39], experimental parasitology [40, 41], parasite identification [31, 35, 42] and host pathology $[43,44]$ research. This is a particularly sensitive issue aimed at minimizing the harm to wildlife, when only a few parasitized individuals could be caged or euthanized if essentially necessary for collection of pathogens, and all other sampled animals can be quickly released according to permit requirements at study sites. In order to optimize and facilitate the diagnostics of blood parasites, a concentration technique was developed for application in parasitology research, the buffy coat method (BCM). The method is based on blood centrifugation and the resulting separation of blood cells and parasites in different layers [45]. It has been commonly used in parasitology, particularly for detection of Trypanosoma species and microfilariae in humans [46-48] and domestic animals [49-52]. A similar technique was also used to diagnose apicomplexan parasites in humans such as Babesia spp. [53] and Plasmodium spp. [54-57]. However, there are few reports on the application of BCM in detection of parasites in wild birds [9, 58-61]. The first application of BCM for detection of bird blood parasites was described by Bennett [58]. Yet, according to his protocol, it was necessary to stain the preparations after blood centrifugation in order to distinguish the parasites. Bennett [58] recognised that he was able to detect Haemoproteus and Leucocytozoon infections in wild birds using BCM, but this technique has not been applied broadly for detection of avian haemosporidian parasites.

The main goals of this study were: (i) to modify the $\mathrm{BCM}$ for more practical use in avian blood parasite sampling during fieldwork; (ii) to test the sensitivity and specificity of the BCM in detection of blood parasites in naturally infected birds using non-stained preparations; (iii) to evaluate the effectiveness of ME of the non-stained preparations collected using BCM in comparison to the ME of Giemsa stained blood films.

\section{Methods \\ Field work and sample collection}

Blood samples were collected from 345 birds of 42 species belonging to 20 families and 2 orders (Additional file 1: Table S1) in Ventès Ragas Ornithological Station, Lithuania $\left(55^{\circ} 20^{\prime} 28.1^{\prime \prime} \mathrm{N}, 21^{\circ} 11^{\prime} 25.3^{\prime \prime} \mathrm{E}\right)$ during May 2019. Birds were caught using "Rybachy" type funnel traps, mist nets and "Zigzag" traps. Approximately $50 \mu \mathrm{l}$ of blood was taken by puncturing the brachial vein and collected using heparinized capillary tubes. A few drops of blood were used to prepare blood films, and the remaining blood was maintained in the capillary tubes to be examined using BCM.

Blood films were air-dried using a battery-powered fan, fixed by immersion in absolute methanol for 1-2 s, dried 
at room temperature using a fan and stained using a fast protocol (blood films were stained in 30\% Giemsa solution for $15 \mathrm{~min}$ at temperature of $\sim 20^{\circ} \mathrm{C}$ ) [62]. This protocol provided good results in distinguishing parasites in blood stages.

\section{Microscopical examination}

One stained blood film from each bird was examined using an Olympus CX23 light microscope (Olympus, Tokyo, Japan) by experienced parasitologists. Approximately 10,000 red blood cells were screened at high magnification $(1000 \times)$ in each blood film. Quick staining and ME of blood films is essential as it provides an opportunity for quick examination and therefore the subsequent release of birds that are non-infected or are non-suitable for further studies, minimizing any potential suffering of sampled individuals. Images of reported parasites were prepared using a Zeiss PrimoStar light microscope equipped with an Axiomcam ERc 5s camera (Carl Zeiss MicroImaging $\mathrm{GmbH}$, Jena, Germany).

\section{Buffy coat method}

Each blood sample was examined using the BCM in parallel to ME. The heparinized capillary tubes with blood were sealed with plasticine at one end (Fig. 1a) and then centrifugated in a microhematocrit centrifuge for $5 \mathrm{~min}$ at $10,000 \times \mathrm{rpm}$. Centrifugation was performed 5-10 min after withdrawal of blood from the birds. Next, the capillary tube was placed above a glass slide fixed with plasticine, the buffy coat area (Fig. 1a) was then examined under low magnification $(100 \times)$ that was effective for visualization of motile stages of relatively large parasites (microfilariae and Trypanosoma spp.) (Fig. 1b). Then, the buffy coat wet preparations were prepared. The capillary tube was broken at the site of the red blood cell layer, approximately $1 \mathrm{~mm}$ below the buffy coat layer (Fig. 1c). This was achieved by gently pressing and running the sharp edge of an objective glass slide on the glass capillary tube. The buffy coat with the adjacent plasma were transferred to an objective glass slide using a capillary tube pump (Fig. 1d), followed by gentle mixing and covering with a coverslip (size of $18 \times 18 \mathrm{~mm}$ ) (Fig. 1e). Finally, this wet preparation was left for approximately 1 min to allow the blood cells to settle on the slide, then the entire preparation was examined for the presence of parasites under microscope at $400 \times$ magnification. Parasite images were prepared using a Zeiss PrimoStar light microscope equipped with Axiomcam ERc 5s camera (Carl Zeiss). After the ME, the coverslip was removed and a thin film was prepared on the objective glass slide, the film was dried, fixed, stained and examined as a blood film.
It is important to note that care should be taken while applying the $\mathrm{BCM}$ to guarantee reliable results, especially when many samples are being processed in parallel. It is mandatory that the samples be examined microscopically immediately after centrifugation, otherwise the actively moving parasites (particularly microfilariae and large trypanosomes) can merge in the red blood cell layer or move into the plasma [46], resulting in possible false negative results. Similar care should also be taken with the wet preparations as they can also quickly dry out on the objective glass slides after preparation. It is therefore recommended to keep the preparations in a humid chamber until examination, especially when multiple samples need to be checked in parallel. Buffy coat wet preparations should be carefully prepared in a way to avoid large volumes of red blood cells being transferred to the slides; this preparation should be thin. If many red blood cells are in these preparations, the cells could form clusters, which prevent the observer from properly identifying or detecting parasites present in the samples. It is also necessary to prepare the buffy coat wet preparations carefully in order to avoid any small pieces of glass that might appear in the preparations, which can result from when the glass capillary is broken; such artefacts might prevent an even preparation of blood films and compromise microscopical examination.

\section{Statistical analysis}

Statistical analysis was carried out using the Statistical Package for the Social Sciences (SPSS) for Windows, version 15.0 (SPSS Inc., Chicago, IL, USA). For comparison with $\mathrm{BCM}$, ME was considered as the gold standard method during the statistical analysis. Parasite detection results were compared between these two diagnostic methods, and the sensitivity (probability to get positive results when the infection is present), the specificity (probability to get negative results when the infection is absent), the positive predictive value (proportion of individuals with positive test results that actually have the infection) and the negative predictive value (proportion of individuals with negative test results that actually do not have the infection) were calculated [63], along with their respective $95 \%$ confidence interval $(95 \% \mathrm{CI})$. A $P$-value of $\leq 0.05$ was considered as significant. Cohen's kappa index (к) and its 95\% CI were also calculated to evaluate concordances between BCM and ME methods [64]. The concordance was considered according to Landis \& Koch [64] as follows: poor, $\kappa<0$; light, $\kappa=0-0.20$; fair, $\kappa=0.21-0.40$; moderate, $\kappa=0.41-0.60$; substantial, $\mathrm{K}=0.61-0.80$; and almost perfect, $\mathrm{\kappa}=0.81-1.00$. 

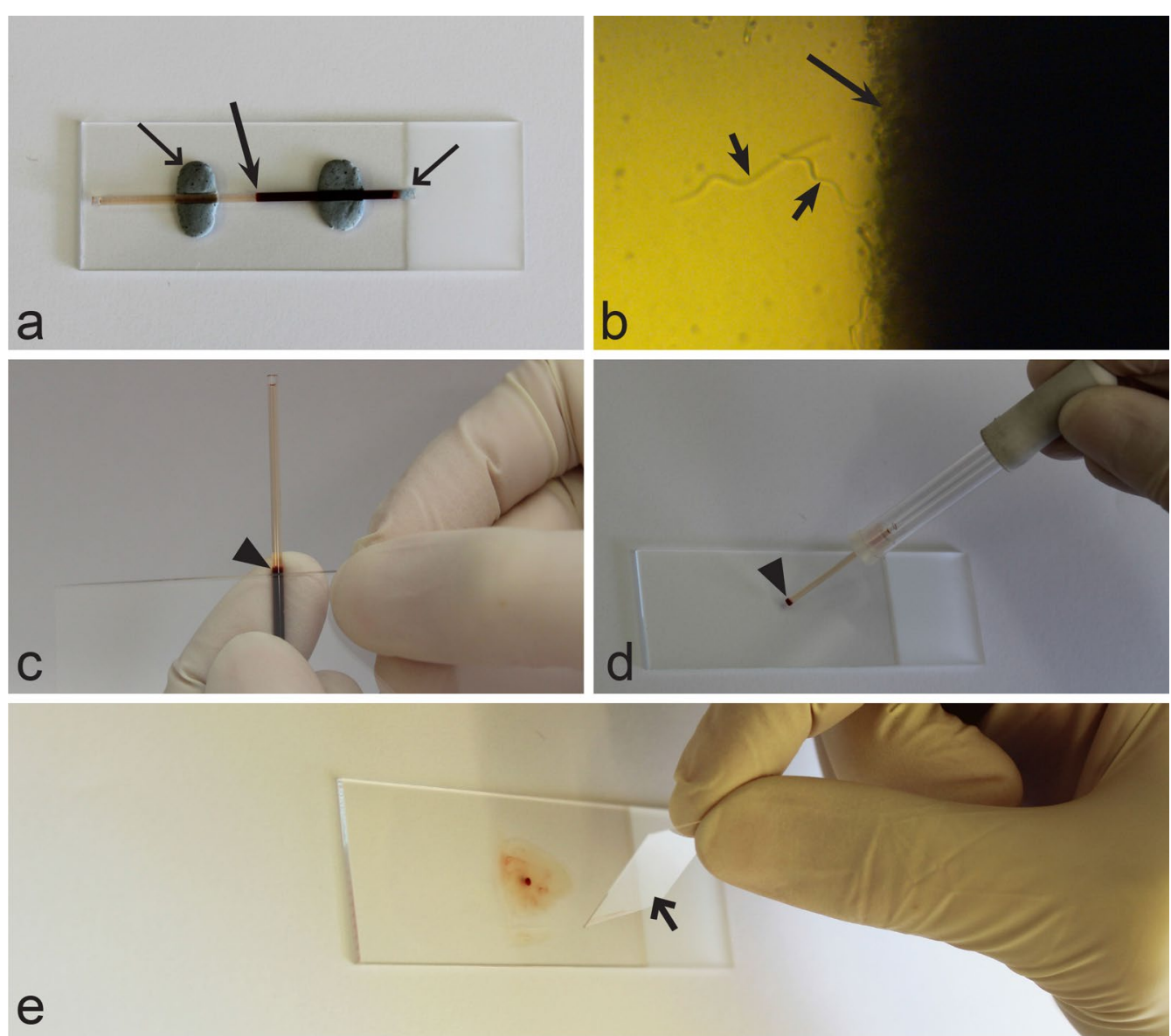

Fig. 1 Main procedures of blood sample preparation for the application of buffy coat method. a Capillary tube with centrifugated blood, which was prepared for initial microscopical examination (note that one tip of the capillary is blocked with plasticine and the entire capillary tube is fixed on the objective glass slide using plasticine). Long barbed arrow, buffy coat; long simple arrow, plasticine. b Buffy coat layer as it looks under light microscope (100x magnification), note that microfilariae are readily visible and locate close to the buffy coat layer (long arrow, buffy coat layer; short arrows, microfilariae). c Capillary tube being broken close to the buffy coat layer using a sharp edge of the objective glass slide (arrowhead, the site where the capillary tube should be broken). $\mathbf{d}$ Blood and the buffy coat layer being transferred to the objective glass slide (arrowhead, buffy coat layer and small portion of red blood cells). e Blood and the buffy coat layer transferred on the objective glass slide and being covered with a coverslip (arrow, cover slip)

\section{Results}

Of the 345 tested samples, 210 (60.9\%) were infected with blood parasites after pooling results of $\mathrm{ME}$ and $\mathrm{BCM}$ testing (Additional file 1: Table S1). In BCM examination, Haemoproteus species were the most prevalent, followed by Trypanosoma, Lankesterella, microfilaria and Leucocytozoon parasites; no Plasmodium infections were detected by this method. In ME, Haemoproteus species were also the most prevalent, followed by Plasmodium, Lankesterella, Leucocytozoon and microfilaria parasites; no Trypanosoma infections were detected by this method (Table 1). Interestingly, BCM could detect approximately twice the number of co-infections than the applied protocol of ME. The most common co-infections detected by BCM were Haemoproteus and Trypanosoma parasites, while the most common co-infection was Haemoproteus and Plasmodium parasites when ME was used (Table 2).

Haemoproteus infections were observed in birds of almost all studied families, while Lankesterella parasites were more prevalent in birds of the Acrocephalidae, with majority of infections seen in the sedge warbler Acrocephalus schoenobaenus. Microfilariae and Leucocytozoon infections, were seen only in birds of the Accipitridae, Fringillidae, Corvidae, Muscicapidae, Phylloscopidae, Scolopacidae and Turdidae, while Trypanosoma species were more common and detected in 23 avian host species belonging to 11 families (Additional file 1: Table S1).

During BCM examination, it was possible to observe parasites moving in the buffy coat wet preparations. Microfilariae were particularly easy to identify even 
Table 1 Prevalence of infection and sensitivity, specificity, positive predictive value (PPV) and negative predictive value (NPV) as well as Cohen's Kappa index (Kappa) based on data obtained using buffy coat method (BCM) and microscopic examination (ME) of blood films

\begin{tabular}{|c|c|c|c|c|c|c|c|c|}
\hline Parasite & Overall prevalence ${ }^{a}$ & $\mathrm{BCM}^{\mathrm{a}}$ & $M E^{a}$ & Sensitivity $^{b}$ & Specificity $^{b}$ & $\mathrm{PPV}^{\mathrm{b}}$ & $N P V^{b}$ & Kappa $^{c}$ \\
\hline Haemoproteus & $135(39.1)$ & 125 (92.6) & $113(83.7)$ & $91.2(85.9-96.4)$ & $90.5(86.8-94.3)$ & $82.4(75.7-89.1)$ & $95.5(92.7-98.2)$ & $0.80(0.73-0.86)$ \\
\hline Trypanosoma & $91(26.4)$ & $91(100)$ & $0(0)$ & $-^{d}$ & $73.6(69.0-78.3)$ & $-^{d}$ & $100(100)$ & $-^{d}$ \\
\hline Plasmodium & $18(5.2)$ & $0(0)$ & $18(100)$ & $--^{\mathrm{d}}$ & $100(100)$ & $-{ }^{d}$ & $94.8(92.4-97.1)$ & $-{ }^{d}$ \\
\hline Lankesterella & $13(3.8)$ & $10(76.9)$ & $7(53.9)$ & $57.1(20.5-93.8)$ & $98.0(97.0-100)$ & $40.0(9.6-70.4)$ & $99.1(98.1-100)$ & $0.46(0.16-0.76)$ \\
\hline Leucocytozoon & $6(1.7)$ & $2(33.3)$ & $5(83.3)$ & $20.0(0-55.1)$ & $99.7(99.1-100)$ & $50.0(0-100)$ & $98.8(97.7-100)$ & $0.28(0-0.72)$ \\
\hline Microfilaria & $6(1.7)$ & $6(100)$ & $1(16.7)$ & $100(100)$ & $98.6(97.3-99.8)$ & $16.7(0-46.5)$ & $100(100)$ & $0.28(0-0.72)$ \\
\hline
\end{tabular}

a Number of positive samples followed by infection prevalence (in \%) in parentheses

b Percentage, followed by the $95 \%$ confidence interval in parentheses

c Kappa index followed by the $95 \%$ confidence interval (in parentheses)

d Calculation not possible because the parasites were detected only by ME

Note: ME was considered as the gold standard method

Table 2 Prevalence of co-infections detected by buffy coat method (BCM) and microscopic examination (ME)

\begin{tabular}{lll}
\hline Co-infection & $\mathrm{BCM}^{\mathrm{a}}$ & $\mathrm{ME}^{\mathrm{a}}$ \\
\hline Haemoproteus + Lankesterella & $2(0.6)$ & $4(1.2)$ \\
Haemoproteus + Leucocytozoon & $1(0.3)$ & $1(0.3)$ \\
Haemoproteus + microfilaria & $2(0.6)$ & $1(0.3)$ \\
Haemoproteus + Plasmodium & - & $7(2.0)$ \\
Haemoproteus + Trypanosoma & $29(8.4)$ & - \\
Microfilaria + Trypanosoma & $1(0.3)$ & - \\
Plasmodium + Trypanosoma & - & $1(0.3)$ \\
Haemoproteus + Lankesterella + Trypanosoma & $4(1.2)$ & - \\
Haemoproteus + Plasmodium + Trypanosoma & - & $3(0.9)$ \\
Total & $39(11.3)$ & $21(6.1)$
\end{tabular}

a Number of positive samples, followed by infection prevalence (in \%) in parentheses

before the buffy coat was transferred to the objective slide due to their large size and fast movement (Figs. 1b, 2c-d). Trypanosoma species were also easy to detect (Fig. 2e, f); however, due to their smaller size they were not so readily visible as microfilaria. The smallest trypomastigotes of the Trypanosoma everetti group are similar in length to red blood cells (Fig. 2g, h). It was possible to distinguish trypanosomes of this group because of their small size and an irregular shape, resembling a leaf or a kite in outline (Fig. 2h) rather than the usual spindle shape associated with trypanosome morphology [65]. Leucocytozoon infections were also detected using BCM, but mainly when relatively large fusiform host-parasite complexes were present (Fig. 2a, b). Haemoproteus spp. were readily observed, but as they are small parasites they were only seen after the buffy coat had been transferred onto objective slides (Fig. 3a, b). It is worth mentioning that mature gametocytes of Haemoproteus parasites start exflagellation a few minutes after exposure to air, and often it was possible to observe not only exflagellation (Fig. 3c, d), but also microgametes (Fig. 3e, f) and even fertilization events and ookinete formation (Fig. 3i, j). In regard to Haemoproteus identification using BCM, it is important to note that macrogametes of the parasites are of similar size as leucocytes, so it is crucial to pay attention to the presence of pigment granules, which are always present in blood stages of haemoproteids, in order to distinguish between these cells (Fig. 3g, h). Some species of Leucocytozoon were seen exflagellating as easy as Haemoproteus spp., but this process was rarely observed in leucocytozoids. Lankesterella infections were readily detected both as intracellular (Fig. 3k, l) and extracellular (Fig. 3m, n) parasites.

A comparison of the results of the ME and BCM diagnostics is shown in Table 1 . Both methods were of a similar high sensitivity and specificity for the detection of Haemoproteus spp. (91.2\% and 90.5\%, respectively) and microfilariae (100\% and $98.6 \%$, respectively) infections. In regard to Lankesterella spp. and Leucocytozoon spp., BCM had a low sensitivity $(57.1 \%$ and $20.0 \%$, respectively) and high specificity (98.2\% and 99.7\%, respectively). Despite of the relatively high specificity for Trypanosoma spp. and Plasmodium spp. in BCM diagnostics, it was not possible to calculate the sensitivity of this tool because these parasites were only found using one method (Table 2).

Cohen's kappa index ( $\mathrm{\kappa}$ ) showed substantial agreement $(\kappa=0.80)$ between BCM and ME (Table 1) for detection of Haemoproteus spp.; moderate agreement $(\kappa=0.46)$ for Lankesterella spp., and fair agreement $(\kappa=0.28)$ for microfilariae and Leucocytozoon spp. It is worth mentioning that although the agreement between these two diagnostic methods was substantial for Haemoproteus 

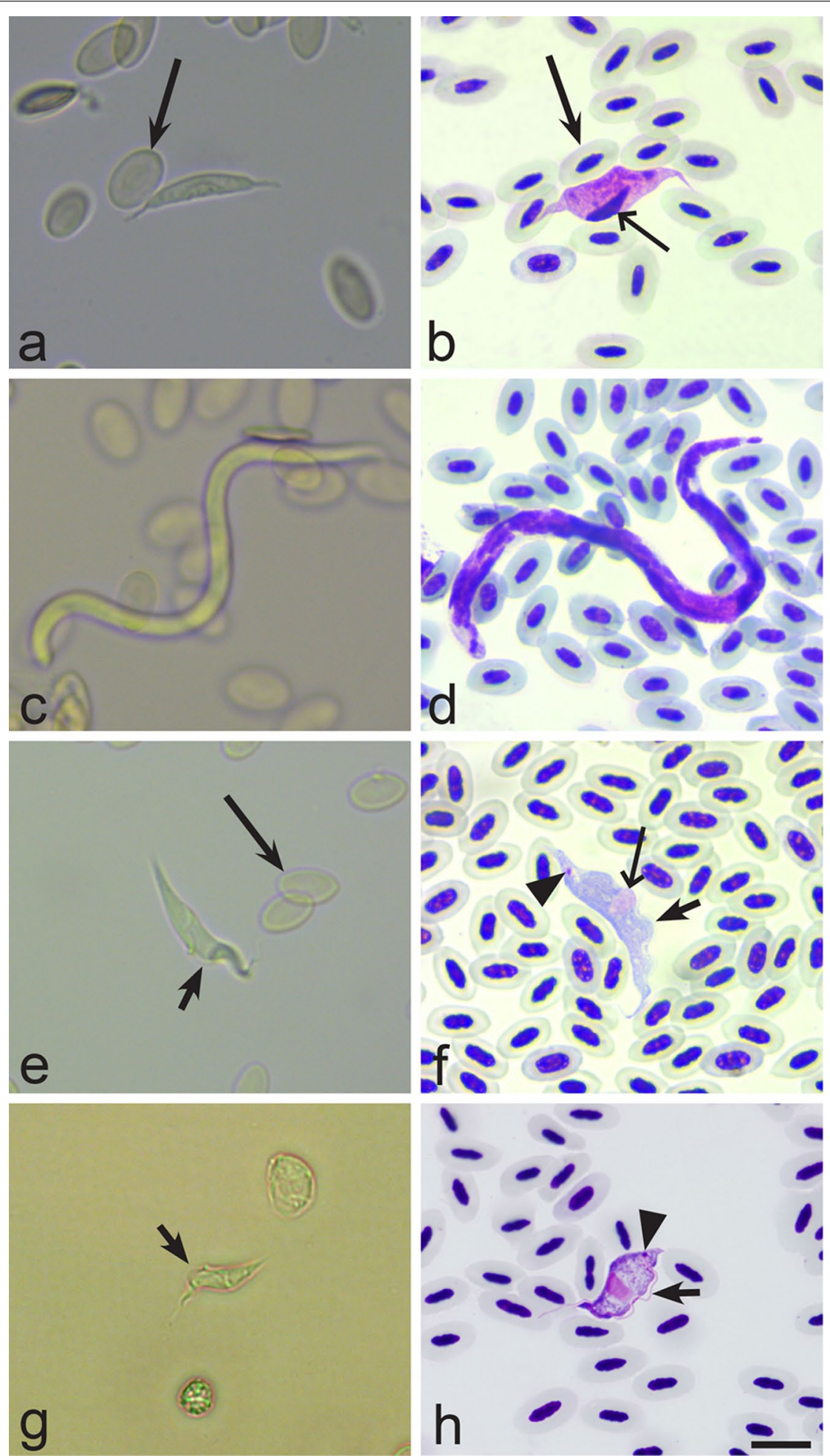

Fig. 2 Images of relatively large blood parasites (larger than red blood cells) showing how they look in the buffy coat wet preparations (a, c, e, $\mathbf{g}$ ) and methanol-fixed Giemsa-stained blood films ( $\mathbf{b}, \mathbf{d}, \mathbf{f}, \mathbf{h})$. $\mathbf{a}, \mathbf{b}$ Leucocytozoon sp. (barbed long arrow, red blood cell; simple long arrow, parasite nucleus). c, d Microfilaria. e, f Trypanosoma sp. (barbed long arrow, red blood cells; short arrow, undulating membrane; arrowhead, kinetoplast; simple long arrow, parasite nucleus). $\mathbf{g}, \mathbf{h}$ Trypanosoma everetti (short arrow, undulating membrane; arrowhead, kinetoplast). Scale-bar: $10 \mu \mathrm{m}$ 
spp., the diagnostic results still did not agree with each other in 32 (9.3\%) of the tested samples. In Lankesterella species diagnostics, which showed the lowest agreement between BCM and ME according to Cohen's kappa index, the results differed in $8(2.3 \%)$ of the tested samples.

\section{Discussion}

The key result of this study is that the BCM is applicable for quick and reliable detection of avian blood parasites belonging to Haemoproteus, Trypanosoma and microfilariae, but is not recommended for Leucocytozoon and Plasmodium species diagnostics. This method could also be used for detection of Lankesterella infections; however, the sensitivity is low. This conclusion is important for fieldwork when infected donor birds should be rapidly screened and selected for parasitological and experimental research. This is a simple and quick parasite detection method, which can be used at each field site using simple microscopes and microcentrifuge equipment.

In addition to methodology results, this study contributes to the knowledge of the prevalence of Haemoproteus, Plasmodium, Leucocytozoon, Lankesterella and Trypanosoma species and microfilariae of filariid nematodes in common European birds (Additional file 1: Table S1). The obtained data corroborate with previous studies that reported prevalence of these blood parasites [66-69]. In the present study, Haemoproteus species had the highest prevalence (Additional file 1: Table S1). It is interesting to note that to date Lankesterella parasites have been reported only in the sedge warbler Acrocephalus schoenoebaenus (Acrocephalidae), Eurasian blue tit Cyanistes caeruleus (Paridae) and snow bunting Plectrophenax nivalis (Calcariidae) $[3,7,70]$. In the present study, the highest prevalence was reported in Acrocephalus birds, but Lankesterella infections were also seen in the lesser whitethroat Sylvia curruca (Sylviidae), European robin Erythacus rubecula (Muscicapidae) and northern wren Troglodytes troglodytes (Troglodytidae). It seems that Lankesterella parasites are more common in European birds than formerly believed; further investigation is needed on this matter.
The main difference between the BCM methods used for detection of blood parasites in humans and other vertebrates and the protocol developed in this study, is that the new protocol does not require any staining procedures, and the parasite diagnostic is possible using only the buffy coat wet preparations. The protocols used in human malariology [71-74] and Trypanosoma spp. diagnostics [46] apply capillary tubes containing acridine orange dye and require fluorescence microscopical examination for parasite visualization. In comparison to the $\mathrm{BCM}$ protocol described in the present study, methods which use fluorescence microscopy are more time consuming and expensive and also with limited application during fieldwork, particularly in remote areas. Additionally, we suggest examination of the buffy coat wet preparations, which is not the case in human malaria and Trypanosoma infection diagnostics. It is interesting that a protocol used in the diagnostics of microfilaria infections in dogs and humans [45] requires a mixture of blood, formalin and methylene blue. Although this protocol applies microscopic analysis of buffy coat wet preparations, all parasites are killed after such fixation.

BCM has a number of advantages in comparison to the gold standard ME. First, this tool does not require staining of blood film preparations thus, is easier to use during fieldwork. Secondly, the avoidance of fixation, drying and staining of blood films reduce all the variables that might result in poor quality of blood films [19]. Thirdly, $\mathrm{BCM}$ is faster than the gold standard ME. On average, BCM diagnostic results for one sample can be obtained in approximately 20 minutes after blood-sampling, while it takes about 40 minutes for the gold standard ME protocol, which was used in this study. If samples from ten birds are examined in parallel, BCM application saves over three hours on diagnostics, which is important during field studies. It should be mentioned that ME used in the present study is relatively fast in comparison to a precise microscopy protocol, which is normally used in blood parasite detection and is recommended in everyday laboratory research $[2,19]$ but is hardly applicable in the field as it is time-consuming (approximately 1.5 hours per sample). Fourthly, BCM gives good results for

\footnotetext{
(See figure on next page.)

Fig. $\mathbf{3}$ Images of relatively small blood parasites (smaller or equal to red blood cells in size) showing how they look in the buffy coat wet preparations ( $\mathbf{a}, \mathbf{c}, \mathbf{e}, \mathbf{g}, \mathbf{i}, \mathbf{k}, \mathbf{m})$ and methanol-fixed Giemsa-stained blood films $(\mathbf{b}, \mathbf{d}, \mathbf{f}, \mathbf{h}, \mathbf{j}, \mathbf{l}, \mathbf{n}) . \mathbf{a}, \mathbf{b}$ Immature gametocytes of Haemoproteus sp. (arrows) inside red blood cells. c, d Haemoproteus sp. microgametocyte during exflagellation, note readily visible microgametes (simple wide arrowheads) still attached to extracellular microgametocytes (barbed arrowhead, pigment granules). e, f Haemoproteus sp. microgametes (simple wide arrowhead). $\mathbf{g}, \mathbf{h}$ Extracellular rounded gametocytes of Haemoproteus sp. (left) and leucocytes (right, barbed long arrow); note that parasite can be readily distinguished from leucocytes due to presence of hemozoin pigment granules (barbed arrowhead). $\mathbf{i}$, $\mathbf{j}$ Numerous Haemoproteus sp. ookinetes at different stages of maturation: immature (triangle wide short arrow), nearly mature and mature parasites (triangle wide long arrow) can be distinguished. Intracellular $(\mathbf{k}, \mathbf{I})$ and extracellular $(\mathbf{m}, \mathbf{n})$ Lankesterella sp. (simple long arrow); note that parasite is closely appressed to nuclei of mononuclear leucocyte (k, I). Scale-bar: $10 \mu \mathrm{m}$
} 


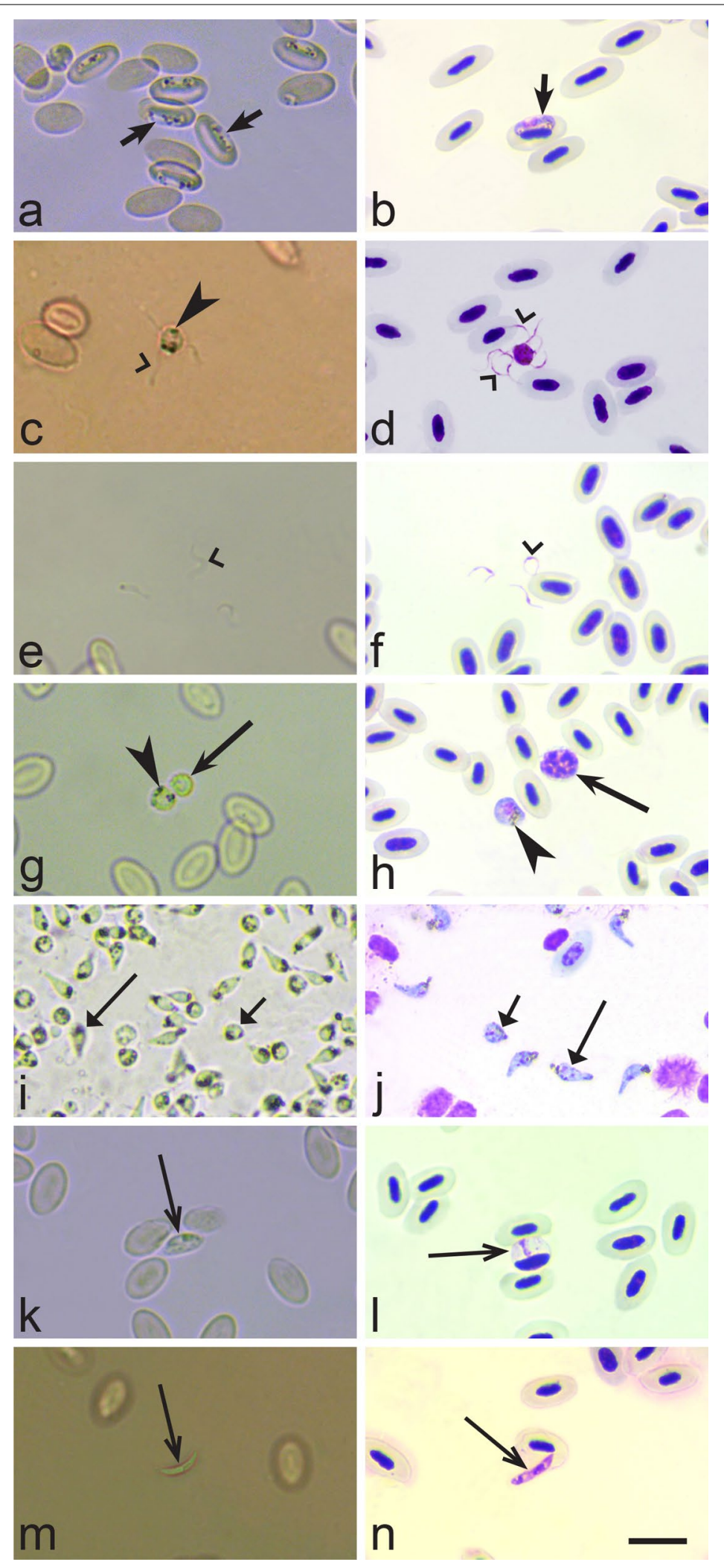


microfilariae and Trypanosoma species detection. The BCM has been known and extensively used in human medicine $[45,47,48,75,76]$. In veterinary medicine, it was mainly applied for examination of mammalian blood samples, with a focus on diagnostics of parasitic infections with zoonotic potential [47, 52, 53, 77-79]. This tool has been insufficiently applied in avian parasitology in wildlife [9, 58-61]. Fifthly, BCM can also be applied to distinguish between Haemoproteus parasites with fast and slow ookinete development since it is readily possible to see ookinetes being formed in the fast-developing species in the buffy coat wet capillary preparation (Fig. 3i). The feature of Haemoproteus parasites to extensively exflagellate quickly (within 10 minutes after infected blood is exposed to air) has been used in haemosporidian genomic research $[40,80]$ as well as gametogenesis and ookinete development studies [2, 81, 82], hybridization experiments [41] and vector studies [62, 83]. Sixthly, $\mathrm{BCM}$ not only leads to a concentration of parasites in one layer but it also uses a larger volume of blood (around 10 times more) than ME, which makes it more sensitive to detect a low parasitaemia [59]. Finally, BCM is more suitable to diagnose co-infections as using this method we were able to detect almost double the number of coinfections than ME (Table 2).

Although $\mathrm{BCM}$ is a promising technique for field research, it has some disadvantages. First, we recommend collecting about $30-50 \mu \mathrm{l}$ of blood for BCM, which might be difficult in the case of small birds, for example the tiny passerine goldcrest Regulus regulus that is common in Europe. Secondly, non-moving parasites can be present both inside and out of the cells, as well as motile stages can occur and should be observed quite rapidly. As a result, some microscopy training might be needed before examination, since these forms are different from those that are seen on blood films stained with Giemsa. This is especially true for young forms of Haemoproteus spp. and Plasmodium spp. (Fig. 3a, b) that are not moving and would be found inside red blood cells as well as Lankesterella parasites (Fig. $3 \mathrm{k}-\mathrm{n}$ ) that move very slowly and are smaller than other blood parasites commonly found in birds. Thirdly, BCM is not recommended to be used for diagnostics of Plasmodium and Leucocytozoon infections. BCM fails to detect Plasmodium infections mainly because malaria parasites do not exflagellate when the blood is simply exposed to air, as is the case in all tested Haemoproteus species [40, 41]. Haemoproteus parasites develop readily visible moving stages (exflagellating microgametocytes and microgametes) in preparations prepared using BCM (Fig. 3d, f). Intracellular blood stages of malaria parasites are often small and difficult to visualize in non-stained preparations [2]. Although the leucocytozoids were seen in a few samples (Table 2), they are often at low intensity and might be difficult to distinguish from leucocytes. In BCM, Leucocytozoon parasites can be readily identified mainly when gametocytes develop in fusiform host cells. Such host-parasite complexes can be readily distinguished from all blood cells (Fig. 2a, b), but it is often difficult to distinguish between leucocytozoids and mononuclear leucocytes when the parasites develop in roundish infected host cells. It is worth noting that although exflagellation of some Leucocytozoon spp. has been described during simple exposure to air [2], this does not seem to happen readily for all Leucocytozoon parasites, as is the case in Haemoproteus species. Absence of pigment granules in Leucocytozoon spp. gametocytes markedly challenges visualization and identification of these parasites during application of the BCM. Fourthly, buffy coat wet capillary preparations can be stained for further microscopical analysis, but we do not recommend using this material to perform any morphological analysis, particularly parasite species description. The deformation of parasites after centrifugation was reported by Bennett [58] and we corroborate these findings. In Haemoproteus parasites, morphological changes might occur not only due to deformation during centrifugation, but also due to changes of mature gametocytes during sexual process and exflagellation that rapidly occur when the blood is exposed to air after withdrawal from birds (Fig. 3i, j). Another possible artefact, which might preclude microscopical examination in stained BCM preparations, might be due to the presence of high amounts of plasma, increasing the amount of protein and resulting in a dark pink staining of the background of the blood film. Despite these disadvantages, BCM is useful for the rapid detection of infected birds, which is essential for parasite detection and for further use in precise studies without harming the avian host.

When choosing a diagnostic method for parasite detection, it is important to known how sensitive and specific it is in comparison to other applied methods [63]. The present study shows that $\mathrm{BCM}$ can be recommended for use in diagnostic detection of Haemoproteus spp. and microfilariae infections, as the method has high sensitivity and specificity for both parasites (Table 2). In regard to Lankesterella infections, it is of relatively low sensitivity, but of high specificity (Table 2). However, BCM had the lowest sensitivity among encountered parasites for Leucocytozoon species despite of its high specificity. In regard to Trypanosoma and Plasmodium infections, we could not measure the BCM sensitivity because these parasites were only detected by one method, i.e. BCM or $\mathrm{ME}$, respectively. These data suggest application of $\mathrm{BCM}$ as a diagnostic method for Trypanosoma infection and ME for Plasmodium infection, respectively. 
It is interesting to note that the agreement between $\mathrm{BCM}$ and ME was considered as substantial in Haemoproteus infection diagnostics according to the Cohen's kappa index (the number of positive samples detected by each of these methods coincided markedly) (Table 1). However, it is always desirable to reduce the time of sample screening during fieldwork, replacing the time-consuming techniques with less time-dependent methods, which certainly is the case with the BCM. The agreement between BCM and ME in detection of other parasites was lower than for Haemoproteus species; however, this study strongly indicates that the former method is markedly more sensitive in the diagnostics of Trypanosoma and microfilariae infections.

Results of diagnostics of human Plasmodium parasites using commercial kits containing capillary tubes with orange acridine were compared with data obtained using microscopical examination of Giemsa-stained thick blood films, which is considered the gold standard diagnose method for human malaria. Reported results were controversial; some studies showed a high sensitivity $[74,84,85]$, while others reported a low sensitivity in the diagnostics $[54,86]$. However, Adeoye \& Nga [54] concluded that this is a useful protocol in early human malaria therapeutic intervention due to minimisation of diagnostic time. The present study corroborates this conclusion, particularly because BCM provides opportunities to analyse samples and select birds for experimental studies more quickly than during ME. Importantly, BCM can also be used for rapid blood parasite diagnostics in veterinary medicine, allowing treatment and/or prophylactic measures to start as soon as possible, with the aim to prevent further spread of disease.

\section{Conclusions}

The quick detection of animals infected with parasites is often an important requirement in wildlife parasitology because it provides opportunities to minimize sampling time, resulting in less harm for individual animals and wildlife populations. That is particularly true in experimental research with avian blood parasites, which need to be selected from wildlife populations, in which infection prevalence is low and many host individuals need to be tested before the appropriate infected animals are selected. This study shows that BCM is a quick, reliable and powerful diagnostics tool, which is recommended to be used for detection of infections in birds, particularly of Haemoproteus, Trypanosoma and microfilariae parasites. This method is cheap, fast and simple to use, and thus is recommended for application during field studies even in remote areas. Importantly, this tool is sensitive for detection of blood parasite co-infections. It could also be applied in studies of blood parasites in other vertebrates, with the aim to increase the speed of diagnosis and to rapidly initiate animal treatment and application of prophylactic measures in disease control.

\section{Supplementary information}

Supplementary information accompanies this paper at https://doi. org/10.1186/s13071-020-3984-8.

Additional file 1: Table S1. Prevalence of blood parasites reported in birds using the buffy coat method (BCM) and microscopic examination (ME) of blood films.

\section{Abbreviations}

BCM: buffy coat method; Cl: confidence interval; $\mathrm{K}$ : Cohen's kappa index; ME: microscopical examination; PCR: polymerase chain reaction.

\section{Acknowledgements}

We thank V. Jusys and V. Eigirdas (Ventès Ragas Ornithological Station, Lithuania) for the valuable partnership during bird catching and Dr. Dovile Bukauskaitè, for assistance during fieldwork and taking some pictures of capillary preparations.

\section{Authors' contributions}

CRFC, RB and GV were involved in the experimental conception and design. CRFC, RB, MI, TI and GV performed the donor bird collection and testing. TI and GV performed blood film microscopic examination. CRFC and RB performed buffy coat microscopic examination. CRFC and GV wrote the manuscript. All authors read and approved the final manuscript.

\section{Funding}

This study was funded by the Research Council of Lithuania (RCL) (No. DOTSUT-137-09.3.3-LMT-K-712-02-0004) and also partly supported by the RCL project S-MIP-17-27 and the Open Access to research infrastructure of the Nature Research Centre under Lithuanian open access network initiative.

\section{Availability of data and materials}

All data generated or analysed during this study are included in this published article and its additional file.

\section{Ethics approval and consent to participate}

This study was performed by licenced researchers. None of the birds suffered apparent injury during sampling, and all birds were released after sampling. The procedures were approved by the Environmental Protection Agency, Vilnius, Lithuania (permit number 2019-04-19 Nr. 23).

\section{Consent for publication}

Not applicable.

\section{Competing interests}

The authors declare that they have no competing interests.

Received: 2 December 2019 Accepted: 18 February 2020

Published online: 27 February 2020

\section{References}

1. Sehgal RN, Jones HI, Smith TB. Molecular evidence for host specificity of parasitic nematode microfilariae in some African rainforest birds. Mol Ecol. 2005;14:3977-88.

2. Valkiūnas G. Avian malaria parasites and other haemosporidian. Boca Raton: CRC Press; 2005

3. Merino S, Martínez J, Martínez-de la Puente J, Criado-Fornelio A, Tomás $\mathrm{G}$, Morales J, et al. Molecular characterization of the $185 \mathrm{rDNA}$ gene of 
an avian Hepatozoon reveals that it is closely related to Lankesterella. J Parasitol. 2006;92:1330-5.

4. Merkel J, Jones HI, Whiteman NK, Gottdenker N, Vargas H, Travis EK, et al. Microfilariae in Galapagos penguins (Spheniscus mendiculus) and flightless cormorants (Phalacrocorax harrisi): genetics, morphology, and prevalence. J Parasitol. 2007:93:495-503.

5. Atkinson CT, Thomas NJ, Hunter DB. Parasitic diseases of wild birds. Oxford: Wiley-Blackwell; 2008.

6. Valkiūnas G, lezhova TA, Carlson JS, Sehgal RN. Two new Trypanosoma species from African birds, with notes on the taxonomy of avian trypanosomes. J Parasitol. 2011;97:924-30.

7. Biedrzycka A, Kloch A, Migalska M, Bielański W. Molecular characterization of putative Hepatozoon sp. from the sedge warbler (Acrocephalus schoenobaenus). Parasitology. 2013;14:695-8.

8. Clark NJ, Wells K, Dimitrov D, Clegg SM. Co-infections and environmental conditions drive the distributions of blood parasites in wild birds. J Anim Ecol. 2016;85:1461-70.

9. Chagas CRF, Gonzalez IHL, Salgado PAB, Ontivero CRGR, Locosque P. Occurrence of endoparasites in Ramphastidae (Aves: Piciformes) in São Paulo Zoo. Helminthologia. 2017;54:81-6.

10. Pornpanom P, Salakij C, Prasopsom P, Lertwatcharasarakul P, Kasorndorkbua C, Santavakul M. Morphological and molecular characterization of avian trypanosomes in raptors from Thailand. Parasitol Res. 2019;118:2419-29.

11. Hayes PM, Lawton SP, Smit NJ, Gibson WC, Davies AJ. Morphological and molecular characterization of a marine fish trypanosome from South Africa, including its development in a leech vector. Parasites Vectors. 2014;7:50.

12. Corrêa LL, Oliveira MSB, Tavares-Dias M, Ceccarelli PS. Infections of Hypostomus spp. by Trypanosoma spp. and leeches: a study of hematology and record of these hirudineans as potential vectors of these hemoflagellates. Rev Bras Parasitol Vet. 2016;25:299-305.

13. Stenberg PL, Bowerman WJ. Hemoparasites in Oregon spotted frogs (Rana pretiosa) from central Oregon, USA. J Wildl Dis. 2008;44:464-8.

14. Bernal XE, Pinto CM. Sexual differences in prevalence of a new species of trypanosome infecting tungara frogs. Int J Parasitol Parasites Wildl. 2016:5:40-7.

15. Telford SR. Hemoparasites of the Reptilia: color atlas and text. Florida: CRC Press; 2009.

16. Samuel WM, Pybus MJ, Kocan AA. Parasitic diseases of wild mammals. London: Manson Publishing; 2001.

17. Jarvi SI, Schultz JJ, Atkinson CT. PCR diagnostics underestimate the prevalence of avian malaria (Plasmodium relictum) in experimentally-infected passerines. J Parasitol. 2002;88:153-8.

18. Durrant KL, Beadell JS, Ishtiaq F, Graves GR, Olson SL, Gering E, et al. Avian haematozoa in South America: a comparison of temperate and tropical zones. Ornithol Monogr. 2016;60:98-111.

19. Valkiūnas $G$, lezhova TA, Križanauskienè A, Palinauskas V, Sehgal RN, Bensch S. A comparative analysis of microscopy and PCR-based detection methods for blood parasites. J Parasitol. 2008;94:1395-401.

20. Dimitrov D, Zehtindjiev P, Bensch S. Genetic diversity of avian blood parasites in SE Europe: cytochrome $b$ lineages of the genera Plasmodium and Haemoproteus (Haemosporida) from Bulgaria. Acta Parasitol. 2010;55:201.

21. Godfrey SS, Nelson NJ, Bull CM. Ecology and dynamics of the blood parasite, Hepatozoon tuatarae (Apicomplexa), in tuatara (Sphenodon punctatus) on Stephens Island, New Zealand. JWild Dis. 2011;47:126-39.

22. Neto JM, Pérez-Rodríguez A, Haase M, Flade M, Bensch S. Prevalence and diversity of Plasmodium and Haemoproteus parasites in the globallythreatened aquatic warbler Acrocephalus paludicola. Parasitology. 2015;142:1183-9.

23. Chagas CR, Valkiūnas G, de Oliveira Guimarães L, Monteiro EF, Guida FJ, Simões RF, et al. Diversity and distribution of avian malaria and related haemosporidian parasites in captive birds from a Brazilian megalopolis. Malar J. 2017:16:83.

24. Kauffman KL, Sparkman A, Bronikowski AM, Palacios MG. Vertical transmission of Hepatozoon in the garter snake Thamnophis elegans. J Wild Dis. 2017;53:121-5.

25. Calil PR, Puorto G, Dunn JC, Chagas CRF, Ramos PL. Molecular and morphological characterization of Hepatozoon spp. in Brazilian snakes. Amphibia-Reptilia. 2019;40:337-47.
26. García-Marquez LJ, Rodríguez-Vázquez A, León- Règagnon V, OsorioSarabia D, García-Preito L. Parasites of Boa constrictor (Squamata: Boidae) captive in Colima, Mexico and their pathological effects. Rev Mex Biodivers. 2019;90:e902687.

27. Rodrigues MS, Lima L, Xavier SCDC, Herrera HM, Rocha FL, Roque ALR et al. Uncovering Trypanosoma spp. diversity of wild mammals by the use of DNA from blood clots. Int J Parasitol Parasites Wildl. 2019:8:171-81.

28. Rosskopf SP, Held J, Gmeiner M, Mordmüller B, Matsiégui PB, Eckerle I, et al. Nycteria and Polychromophilus parasite infections of bats in Central Gabon. Infect Genet Evol. 2019;68:30-4.

29. Teodoro AKM, Cutolo AA, Motoie G, Meira-Strejevitch CDS, Pereira-Chioccola VL, Mendes TMF, et al. Gastrointestinal, skin and blood parasites in Didelphis spp. from urban and sylvatic areas in São Paulo state, Brazil. Vet Parasitol Reg Stud Rep. 2016;16:100286.

30. Bernotienè R, Palinauskas V, lezhova T, Murauskaitè D, Valkiūnas G. Avian haemosporidian parasites (Haemosporida): a comparative analysis of different polymerase chain reaction assays in detection of mixed infections. Exp Parasitol. 2016;163:31-7.

31. Lotta IA, Valkiūnas G, Pacheco MA, Escalante AA, Hernández SR, Matta NE. Disentangling Leucocytozoon parasite diversity in the neotropics: descriptions of two new species and shortcomings of molecular diagnostics for leucocytozoids. Int J Parasitol Parasites Wildl. 2019;9:159-73.

32. Valkiūnas $\mathrm{G}$, Bensch $\mathrm{S}$, lezhova TA, Križanauskienè A, Hellgren O, Bolshakov CV. Nested cytochrome $b$ polymerase chain reaction diagnostics underestimate mixed infections of avian blood haemosporidian parasites: microscopy is still essential. J Parasitol. 2006;92:418-22.

33. Martínez J, Martínez-de La Puente J, Herrero J, Del Cerro S, Lobato E, Rivero-de Aguilar J, et al. A restriction site to differentiate Plasmodium and Haemoproteus infections in birds: on the inefficiency of general primers for detection of mixed infections. Parasitology. 2009;136:713-22.

34. Santiago-Alarcon D, Rodríguez-Ferraro A, Parker PG, Ricklefs RE. Different meal, same flavor: cospeciation and host switching of haemosporidian parasites in some non-passerine birds. Parasites Vectors. 2014;7:286.

35. Mantilla JS, González AD, Lotta IA, Moens M, Pacheco MA, Escalante AA, et al. Haemoproteus erythrogravidus n. sp. (Haemosporida, Haemoproteidae): description and molecular characterization of a widespread blood parasites of birds in South America. Acta Trop. 2016;159:83-94.

36. Bukauskaitè D, lezhova TA, Ilgūnas M, Valkiūnas G. High susceptibility of the laboratory-reared biting midges Culicoides nubeculosus to Haemoproteus infections, with review on Culicoides species that transmit avian haemoproteids. Parasitology. 2018;146:333-41.

37. Harl J, Himmel T, Valkiūnas G, Weissenböck H. The nuclear 185 ribosomal DNAs of avian haemosporidian parasites. Malar J. 2019;18:305.

38. Humphries MB, Stacy MT, Ricklefs RE. Population structure of avian malaria parasites. Ecol Evol. 2019;9:7741-51.

39. Huang YL, Tsai SS, Thongchan D, Khatri-Chhetri R, Wu HY. Filarial nematode infection in eclectus parrots (Eclectus roratus) in Taiwan. Avian Pathol. 2017:46:188-94.

40. Palinauskas V, Križanauskienè A, lezhova $T A$, Bolshakov CV, Jönsson J, Bensch S, et al. A new method for isolation of purified genomic DNA from haemosporidian parasites inhabiting nucleated red blood cells. Exp Parasitol. 2013;133:275-80.

41. Valkiūnas G, Palinauskas V, Križanauskienè A, Bernotienè R, Kazlauskienè R, lezhova TA. Further observations on in vitro hybridization of hemosporidian parasites: patterns of ookinete development in Haemoproteus spp. J Parasitol. 2013;99:124-36.

42. Chagas CRF, Bukauskaitè D, llgūnas M, lezhova T, Valkiūnas $G$. A new blood parasite of leaf warblers: molecular characterization, phylogenetic relationships, description and identification of vectors. Parasites Vectors. 2018:11:538

43. Taunde PA, Bianchi MV, Perles L, da Silva FS, Guim TN, Stadler RA, et al. Pathological and molecular characterization of avian malaria in captive Magellanic penguins (Spheniscus magellanicus) in South America. Parasitol Res. 2019;118:599-606.

44. Ilgūnas M, Chagas CRF, Bukauskaitè D, Bernotienè R, lezhova T, Valkiūnas $\mathrm{G}$. The life-cycle of the avian haemosporidian parasite Haemoproteus majoris, with emphasis on the exoerythrocytic and sporogonic development. Parasites Vectors. 2019;12:516.

45. Knott J. A method for making microfilarial surveys on day blood. Trans R Soc Trop Med Hyg. 1939;33:191-6. 
46. Bailey JW, Smith DH. The quantitative buffy coat for the diagnosis of trypanosomes. Trop Dr. 1994;24:54-6.

47. Bouteille B, Buguet A. The detection and treatment of human African trypanosomiasis. Res Rep Trop Med. 2012;3:35-45.

48. Ngoyi DM, Ekangu RA, Kodi MFM, Pyana PP, Balharbi F, Decq M, et al. Performance of parasitological and molecular techniques for the diagnosis and surveillance of gambiense sleeping sickness. PLoS Negl Trop Dis. 2014;8:e2954.

49. Newton W, Wright W. The occurrence of a dog filariid other than Dirofilaria immitis in the United States. J Parasitol. 1956;42:246-58.

50. Wang LC. Evaluation of quantitative buffy coat analysis in the detection of canine Dirofilaria immitis infection: a model to determine its effectiveness in the diagnosis of human filariasis. Parasitol Res. 1998;4:246-8.

51. Mylonakis ME, Papadopoulos E, Koutinas AF, Paitaki C, Leontides L. Comparative methodology for the detection and differentiation of circulating microfilariae of Dirofilaria immitis in the dog. J Helminthol. 2004;78:137-40.

52. Marcos R, Pereira C, Santos M, Luzzago C, Lauzi S, Maia JP, et al. Buffy coat smear or Knott's test: which to choose for canine microfilaria screening in field studies? Vet Clin Pathol. 2016;45:201-5.

53. Mattia AR, Waldron MA, Sierra LS. Use of the quantitative buffy coat system for detection of parasitemia in patients with babesiosis. J Clin Microbiol. 1993;31:2816-8.

54. Adeoye GO, Nga IC. Comparison of quantitative buffy coat technique (QBC) with Giemsa-stained thick film (GTF) for diagnosis of malaria. Parasitol Int. 2007;56:308-12.

55. Kocharekar MM, Sarkar SS, Dasgupta D. Comparative study of modified quantitative buffy coat and two rapid tests in comparison with peripheral blood smear in malaria diagnosis in Mumbai, India. J Parasitol Res. 2014. https://doi.org/10.1155/2014/194651.

56. Ifeorah IK, Brown BJ, Sodeinde OO. A comparison of rapid diagnostic testing (by Plasmodium lactate dehydrogenase), and quantitative buffy coat technique in malaria diagnosis in children. Afr J Infect Dis. 2017;11:31-8

57. Charpentier E, Benichou E, Pagès A, Chauvin P, Fillaux J, Valentin A, et al. Performance evaluation of different strategies based on microscopy techniques, rapid diagnostic test and molecular loop-mediated isothermal amplification assay for the diagnosis of imported malaria. Clin Microbiol Infect. 2020;26:115-21.

58. Bennett GF. The haematocrit for laboratory diagnosis of hematozoa. Can J Zool. 1962;40:124-5.

59. Seegar WS. Comparison of four blood survey techniques for detecting microfilariae in avian blood. Int J Avian Sci. 1978;121:104-6.

60. Hamer GL, Anderson TK, Berry GE, Makohon-Moore AP, Crafton JC, Brawn JD, et al. Prevalence of filarioid nematodes and trypanosomes in American robins and house sparrows, Chicago USA. Int J Parasitol Parasites Wildl. 2012;2:42-9.

61. Atawal AF, Mgbeahuruike AC, Hammers M. Microfilarial infections associated with body mass loss of village weavers Ploceus cucullatus. Ostrich. 2019;90:41-4.

62. Bukauskaitè D, Chagas CRF, Bernotienè R, Žiegytè R, Ilgūnas M, lezhova T, et al. A new methodology for sporogony research of avian haemoproteids in laboratory-reared Culicoides spp., with description of complete sporogonic development of Haemoproteus pastoris. Parasites Vectors. 2019;12:582.

63. Akobeng AK. Understanding diagnostic tests 1: sensitivity, specificity and predictive values. Acta Paediatr. 2007;96:338-41.

64. Landis JR, Koch GG. The measurement of observer agreement for categorical data. Biometrics. 1977;33:159-74.

65. Molyneux DH. Trypanosoma everetti sp. nov. A trypanosome from the black-rumped waxbill Estrilda t. troglodytes Lichtenstein. Ann Trop Med Parasitol. 1973;67:219-22

66. Shurulinkov P, Golemansky V. Plasmodium and Leucocytozoon (Sporozoa: Haemosporida) of wild birds in Bulgaria. Acta Protozool. 2003;42:205-14.

67. Clark NJ, Clegg SM, Lima MR. A review of global diversity in avian haemosporidians (Plasmodium and Haemoproteus: Haemosporida): new insights from molecular data. Int J Parasitol. 2014;44:329-38.

68. Dunn JC, Stockdale JE, Bradford EL, McCubbin A, Morris AJ, Grice PV, et al. High rates of infection by blood parasites during the nestling phase in UK columbids with notes on ecological associations. Parasitology. 2017;144:622-8.

69. Schumm YR, Wecker C, Marek C, Wassmuth M, Bentele A, Willems H, et al. Blood parasites in Passeriformes in central Germany: prevalence and lineage diversity of Haemosporida (Haemoproteus, Plasmodium and Leucocytozoon) in six common songbirds. PeerJ. 2019;6:e6259.

70. Martínez J, Merino S, Badás EP, Almazán L, Moksnes A, Barbosa A Hemoparasites and immunological parameters in Snow Bunting (Plectrophenax nivalis) nestlings. Polar Biol. 2018;41:1855-66.

71. Baird JK, Purnomo P, Jones TR. Diagnosis of malaria in the field by fluorescence microscopy of QBC capillary tubes. Trans R Soc Trop Med Hyg. 1992;86:3-5.

72. Petersen E, Marbiah NT. QBC and thick blood films for malaria diagnosis under field conditions. Trans R Soc Trop Med Hyg. 1994;88:416-7.

73. Vaidya KA, Sukesh K. Quantitative buffy coat (QBC) test and other diagnostic techniques for diagnosing malaria: review of literature. Natl J Med Res. 2012;2:386-8.

74. Benito A, Roche J, Molina R, Amela C, Alvar J. Application and evaluation of QBC malaria diagnosis in a holoendemic area. Appl Parasitol. 1994;35:266-72.

75. Vera-Ku M, Meza-González G, Carlier Y, Truyens C, Gamboa-León R. Comparison of methodologies for detecting Trypanosoma cruzi parasites by microscopic observation of microhematocrit capillary tubes. Rev Soc Bras Med Trop. 2019;52:e20180505.

76. Woo PT. Evaluation of the haematocrit centrifuge and other techniques for the field diagnosis of human trypanosomiasis and filariasis. Acta Trop. 1971;28:298-303.

77. Njenga SM, Wamae CN. Evaluation of ICT filariasis card test using whole capillary blood: comparison with Knott's concentration and counting chamber methods. J Parasitol. 2001;87:1140-3.

78. Ruiz-Piña HA, Cruz-Reyes A. The opossum Didelphis virginiana as a synanthropic reservoir of Trypanosoma cruzi in Dzidzilché, Yucatán, México. Mem Inst Oswaldo Cruz. 2002;97:613-20.

79. Simo G, Njitchouang GR, Njiokou F, Cuny G, Asonganyi T. Genetic characterization of Trypanosoma brucei circulating in domestic animals of the Fontem sleeping sickness of Cameroon. Microbes Infect. 2012;14:651-8.

80. Videvall E. Genomic advances in avian malaria research. Trends Parasitol. 2019;35:254-66.

81. Dimitrov D, Valkiūnas G, Zehtindjiev P, Ilieva M, Bensch S. Molecular characterization of haemosporidian parasites (Haemosporida) in yellow wagtail (Motacilla flava), with description of in vitro ookinetes of Haemoproteus motacillae. Zootaxa. 2013;3666:369-81.

82. Coral AA, Valkiūnas G, González AD, Matta NE. In vitro development of Haemoproteus columbae (Haemosporida: Haemoproteidae), with perspectives for genomic studies of avian haemosporidian parasites. Exp Parasitol. 2015;157:163-9.

83. Chagas CRF, Bukauskaite D, Ilgūnas $M$, Bernotienè R, lezhova T, Valkiūnas G. Sporogony of four Haemoproteus species (Haemosporida: Haemoproteidae), with report of in vitro ookinetes of Haemoproteus hirundinis: phylogenetic inference indicates patterns of haemosporidian parasite ookinete development. Parasites Vectors. 2019;12:422.

84. Gurung B, Bairy I, Jagadishchandra J, Manohar C. Evaluation of falcivax against quantitative buffy coat (QBC) for the diagnosis of malaria. Int J Coll Res Inter Med Public Health. 2010;2:132-40.

85. Bhandari P, Raghuveer C, Rajeev A, Bhandari P. Comparative study of peripheral blood smear, quantitative buffy coat and modified centrifuged blood smear in malaria diagnosis. Indian J Pathol Microbiol. 2008;51:108-12.

86. Salako LA, Akinyanju O, Afolabi BM. Comparison of the standard Giemsastained thick blood smear with the quantitative buffy coat technique in malaria diagnosis in Nigeria. Niger Q J Hosp Med. 1999;9:256-69.

\section{Publisher's Note}

Springer Nature remains neutral with regard to jurisdictional claims in published maps and institutional affiliations. 